\title{
Note on Dates and Transliteration
}

All dates refer to the Old Style (Julian) calendar in use in Russia until February 1918.

The system of transliteration used here is the Library of Congress system. Names and places that are well known are presented in their more familiar English form. 
